\title{
MAXIMA AND HIGH LEVEL EXCURSIONS OF STATIONARY GAUSSIAN PROCESSES ${ }^{1}$ )
}

BY

\author{
SIMEON M. BERMAN
}

\begin{abstract}
Let $X(t), t \geqq 0$, be a stationary Gaussian process with mean 0 , variance 1 and covariance function $r(t)$. The sample functions are assumed to be continuous on every interval. Let $r(t)$ be continuous and nonperiodic. Suppose that there exists $\alpha$, $0<\alpha \leqq 2$, and a continuous, increasing function $g(t), t \geqq 0$, satisfying
\end{abstract}

$$
\lim _{t \rightarrow 0} \frac{g(c t)}{g(t)}=1, \text { for every } c>0,
$$

such that

$$
1-r(t) \sim g(|t|)|t|^{\alpha}, \quad t \rightarrow 0 .
$$

For $u>0$, let $v$ be defined (in terms of $u$ ) as the unique solution of

$$
u^{2} g(1 / v) v^{-\alpha}=1 \text {. }
$$

Let $I_{A}$ be the indicator of the event $A$; then

$$
\int_{0}^{T} I_{[X(s)>u]} d s
$$

represents the time spent above $u$ by $X(s), 0 \leqq s \leqq T$. It is shown that the conditional distribution of

$$
v \int_{0}^{T} I_{[X(s)>u]} d s,
$$

given that it is positive, converges for fixed $T$ and $u \rightarrow \infty$ to a limiting distribution $\Psi_{\alpha}$, which depends only on $\alpha$ but not on $T$ or $g$.

Let $F(\lambda)$ be the spectral distribution function corresponding to $r(t)$. Let $F^{(p)}(\lambda)$ be the iterated $p$-fold convolution of $F(\lambda)$. If, in addition to $(0.2)$, it is assumed that

$$
F^{(p)} \text { is absolutely continuous for some } p>0,
$$

then $\max (X(s): 0 \leqq s \leqq t)$, properly normalized, has, for $t \rightarrow \infty$, the limiting extreme value distribution $\exp \left(-e^{-x}\right)$.

Received by the editors September 22, 1970.

AMS 1970 subject classifications. Primary 60G10, 60G15, 60G17; Secondary 60F99.

Key words and phrases. Conditional distribution, limiting distribution, extreme value distribution, asymptotic independence, excursion over high level, sample function maximum, occupation time, correlation function, local behavior, spectral distribution, absolute continuity, method of moments, Laplace-Stieltjes transform.

(1) This paper represents results obtained at the Courant Institute of Mathematical Sciences, New York University, under the sponsorship of the National Science Foundation, Grant NSF-GP-11460.

Copyright (C) 1971, American Mathematical Society 
If, in addition to (0.2), it is assumed that $F(\lambda)$ is absolutely continuous with the derivative $f(\lambda)$,

and

$$
\lim _{h \rightarrow 0} \log h \int_{-\infty}^{\infty}|f(\lambda+h)-f(\lambda)| d \lambda=0,
$$

then (0.4) has, for $u \rightarrow \infty$ and $T \rightarrow \infty$, a limiting distribution whose Laplace-Stieltjes transform is

$$
\exp \left[\text { constant } \int_{0}^{\infty}\left(e^{-\lambda x}-1\right) d \Psi_{\alpha}(x)\right], \quad \lambda>0 .
$$

1. Discussion of the results. The conditional limiting distribution of (0.4) for fixed $T$ was obtained for $\alpha=2$ and $g=$ constant in [3], and for $\alpha=1$ and $g=$ constant in [5]. We remark that when $\alpha=2$ then the only nontrivial case is $g \equiv$ constant; indeed, $2 t^{-2}(1-r(t))$ converges for $t \rightarrow 0$ to $\int_{-\infty}^{\infty} \lambda^{2} d F(\lambda)$ which is either 0 , positive or infinite. If $g(0)=0$, then the first case arises and $X(t) \equiv X(0)$, so that $r(t) \equiv 1$; therefore, the only interesting case is $g(0)>0$. If $\alpha<2$, then $g(0)=0$ is possible; for example, if the spectral density $f(\lambda)$ exists and

$$
f(\lambda)=|\lambda|^{-1-\alpha} g\left(|\lambda|^{-1}\right) \text { for large }|\lambda|,
$$

then (0.2) is satisfied for some constant multiple of $g(|t|)$.

The extreme value limit distribution has been obtained for $\alpha=2$ in several works under increasingly general conditions (Volkonskiǐ and Rozanov [14], Cramér and Leadbetter [6], Beljaev [1], Qualls [11], and Berman [4]). For $\alpha=1$ and $g$ constant, it was studied by Pickands [10]. A sufficient condition for (0.5) is

$$
\int_{-\infty}^{\infty}|r(s)|^{p} d s<\infty \text { for some } p>0
$$

indeed, if $p$ is an integer, $r^{p}(s)$ is the Fourier-Stieltjes transform of $F^{(p)}$. (1.1) is implied by several of the conditions used in earlier works, namely, $r(t)=O\left(t^{-\varepsilon}\right)$, $t \rightarrow \infty$ for some $\varepsilon>0$ (see [6, p. 257]) and the integrability of $r^{2}$ (see [4] and [10]).

The only previous investigation of the limiting distribution of $(0.4)$ for $u \rightarrow \infty$ and $T \rightarrow \infty$ is that of Volkonskii and Rozanov [14] in the case $\alpha=2$. In addition to (0.2) they also assumed that $1-r(t)-g(0) t^{2} \sim \lambda_{4} t^{4} / 4$ !; and, in place of the mild conditions (0.6) and (0.7), assumed that the process satisfies the "strong mixing condition." Their proof is based on the fact that the "horizontal-window" conditional limiting distribution of the excursion above a high level is $\Psi_{2}$, the Rayleigh distribution; and that the upcrossings tend to a limiting Poisson process.

Our condition (0.6) implies that $r(t) \rightarrow 0$ for $t \rightarrow \infty$ (Riemann-Lebesgue Theorem). It also implies that $\int_{-\infty}^{\infty}|f(\lambda+h)-f(\lambda)| d \lambda$ tends to 0 with $h$. The condition (0.7) prescribes a rate for such convergence. It is well known that this rate is related to the rate of convergence of $r(t)$ to 0 for $t \rightarrow \infty$ (see [13]). 
2. Limiting conditional distribution of a high level excursion. Let $U(t), t \geqq 0$, be a Gaussian process with continuous sample functions, and with the specific moment functions

$$
E U(t) \equiv 0, \quad E U^{2}(0)=0, \quad E|U(t)-U(s)|^{2}=2|t-s|^{\alpha} .
$$

Put $\phi(u)=(2 \pi)^{-1 / 2} e^{-x^{2} / 2}$. Let $X(t)$ be a general process satisfying the conditions leading to and including (0.2).

THEOREM 2.1. Under condition (0.2), for every $T>0$ and $m \geqq 1$,

$$
\lim _{u \rightarrow \infty} \frac{E\left[v \int_{0}^{T} I_{[X(s)>u]} d s\right]^{m}}{T v \phi(u) / u}=m \int_{0}^{\infty} E\left\{\int_{0}^{\infty} I_{\left[U(s)>s^{\alpha}-z\right]} d s\right\}^{m-1} e^{-z} d z .
$$

Proof. The expectation in the numerator of (2.1) is equal to

$$
E\left\{\int_{0}^{T v} I_{[X(s / v)>u]} d s\right\}^{m}=m \int_{0}^{T v} \int_{0}^{s_{m}} \cdots \int_{0}^{s_{m}} P\left\{X\left(\frac{s_{i}}{v}\right)>u, i=1, \ldots, m\right\} d s_{1} \cdots d s_{m},
$$

which, by stationarity, is equal to

$$
m \int_{0}^{T v} \int_{0}^{s_{m}} \cdots \int_{0}^{s_{m}} P\left\{X\left(\frac{s_{m}-s_{i}}{v}\right)>u, i=1, \ldots, m-1, X(0)>u\right\} d s_{1} \cdots d s_{m} .
$$

This is equal to

$$
m \int_{0}^{T v} \int_{0}^{s_{m}} \cdots \int_{0}^{s_{m}} P\left\{X\left(\frac{s_{i}}{v}\right)>u, i=1, \ldots, m-1, X(0)>u\right\} d s_{1} \cdots d s_{m}
$$

because, for any function $f$,

$$
\int_{0}^{t} f(t-s) d s=\int_{0}^{t} f(s) d s .
$$

By the total probability formula, the integrand in (2.2) is equal to

$$
\int_{u}^{\infty} P\left\{X\left(\frac{s_{i}}{v}\right)>u, i=1, \ldots, m-1 \mid X(0)=y\right\} \phi(y) d y,
$$

which, by the substitution $y=u+z / u$ and the identity

$$
\phi(u+z / u)=\phi(u) e^{-z} \exp \left(-z^{2} / 2 u^{2}\right)
$$

is equal to

$$
\frac{\phi(u)}{u} \int_{0}^{\infty} P\left\{X\left(\frac{s_{i}}{v}\right)>u, i=1, \ldots, m-1 \mid X(0)=u+\frac{z}{u}\right\} e^{-z} \exp \left(-z^{2} / 2 u^{2}\right) d z .
$$

We evaluate the conditional probability in (2.3). The process $u[X(s / v)-u]$ is conditionally Gaussian, given $X(0)=u+z / u$, with conditional mean

$$
u^{2}[r(s / v)-1]+z r(s / v)
$$

and conditional covariance (for $0<s<t$ )

$$
u^{2}[r((t-s) / v)-r(s / v) r(t / v)]
$$


By $(0.1),(0.2)$ and $(0.3)$, the conditional mean converges to $-s^{\alpha}+z$ and the conditional covariance to $s^{\alpha}+t^{\alpha}-(t-s)^{\alpha}$. These are the mean and covariance, respectively, of the process $U(s)-s^{\alpha}+z$; thus, the process $u[X(s / v)-u]$ converges in conditional distribution to the process $U(s)-s^{\alpha}+z$. It follows that the integrand in (2.3) converges everywhere to

$$
P\left\{U\left(s_{i}\right)>s_{i}^{\alpha}-z, i=1, \ldots, m-1\right\} e^{-z} .
$$

We substitute (2.3) for the integrand in (2.2), divide by $T v \phi(u) / u$, and formally pass to the limit under the sign of integration to get

$$
m \int_{0}^{\infty} \cdots \int_{0}^{\infty} P\left\{U\left(s_{i}\right)>s_{i}^{\alpha}-z, i=1, \ldots, m-1\right\} e^{-z} d z d s_{1} \cdots d s_{m-1},
$$

which is equal to the right-hand side of (2.1).

The inversion of the limit operation has to be justified. The integrand in (2.2) does not depend on the variable $s_{m}$; therefore, the integral is equal to

$$
\begin{aligned}
m \int_{0}^{T v}\left(T v-s_{m-1}\right) & \int_{0}^{s_{m-1}} \\
& \cdots \int_{0}^{s_{m-1}} P\left\{X\left(\frac{s_{i}}{v}\right)>u, i=1, \ldots, m-1, X(0)>u\right\} d s_{1} \cdots d s_{m-1},
\end{aligned}
$$

which, when divided by $T v \phi(u) / u$ and transformed as was (2.3), is

$$
\begin{array}{r}
m \int_{0}^{T v}\left(1-\frac{s_{m-1}}{T v}\right) \int_{0}^{s_{m-1}} \cdots \int_{0}^{s_{m-1}} \int_{0}^{\infty} P\left\{X\left(\frac{s_{i}}{v}\right)>u, i=1, \ldots, m-1 \mid X(0)=u+\frac{z}{u}\right\} \\
\cdot e^{-z} \exp \left(-z^{2} / 2 u^{2}\right) d z d s_{1} \cdots d s_{m-1}
\end{array}
$$

If $Y$ has a Gaussian distribution with mean $\mu$ and variance $\sigma^{2}$, then

$$
P\{Y>u\} \leqq e^{-c u} E e^{c Y}=\exp \left(-c u+c \mu+\frac{1}{2} c^{2} \sigma^{2}\right), \quad c>0 ;
$$

furthermore, if $X\left(s_{i} / v\right)>u$ for $i=1, \ldots, m-1$, then

$$
\frac{u}{m-1} \sum_{i=1}^{m-1}\left[X\left(s_{i} / v\right)-u\right]>0 ;
$$

thus, by (2.4) and (2.5),

$$
\begin{aligned}
P\left\{X\left(s_{i} / v\right)>\right. & u, i=1, \ldots, m-1|X(0)=u+z| u\} \\
\leqq & P\left\{\frac{u}{m-1} \sum_{i=1}^{m-1}\left[X\left(\frac{s_{i}}{v}\right)-u|>0| X(0)=u+\frac{z}{u}\right\}\right. \\
\leqq & \exp \left\{c u^{2}\left[\frac{1}{m-1} \sum_{i=1}^{m-1}\left(r\left(\frac{s_{i}}{v}\right)-1\right)+\frac{c z}{m-1} \sum_{i=1}^{m-1} r\left(\frac{s_{i}}{v}\right)\right]\right\} \\
& \cdot \exp \left\{\frac{c^{2}}{2} \cdot \operatorname{Var}\left[\frac{u}{m-1} \sum_{i=1}^{m-1}\left(X\left(\frac{s_{i}}{v}\right)-u\right) \mid X(0)\right]\right\} .
\end{aligned}
$$


Since the variance is unchanged by an additive constant, and since the conditional variance never exceeds the unconditional, we have

$$
\begin{aligned}
\operatorname{Var}\left\{\frac { u } { m - 1 } \sum _ { i = 1 } ^ { m - 1 } \left(X\left(\frac{s_{i}}{v}\right)\right.\right. & -u) \mid X(0)\} \\
& =\operatorname{Var}\left\{\frac{u}{m-1} \sum_{i=1}^{m-1}\left(X\left(\frac{s_{i}}{v}\right)-X(0)\right) \mid X(0)\right\} \\
& \leqq E\left\{\frac{u}{m-1} \sum_{i=1}^{m-1}\left(X\left(\frac{s_{i}}{v}\right)-X(0)\right)\right\}^{2} \\
& \leqq E\left\{\frac{u^{2}}{m-1} \sum_{i=1}^{m-1}\left(X\left(\frac{s_{i}}{v}\right)-X(0)\right)^{2}\right\}=\frac{2 u^{2}}{m-1} \sum_{i=1}^{m-1}\left[1-r\left(\frac{s_{i}}{v}\right)\right] .
\end{aligned}
$$

It follows that the last member of $(2.8)$ is at most equal to

$$
\exp \left[c z-c(1-c) \frac{u^{2}}{m-1} \sum_{i=1}^{m-1}\left[1-r\left(\frac{s_{i}}{v}\right)\right]\right]
$$

for $0<c<1$. Under (0.1) and (0.2) there exists, for $T>0$, a constant $B>0$ such that

$$
1-r(t) \geqq B g(t)|t|^{\alpha}, \quad 0 \leqq t \leqq T ;
$$

therefore, by (0.3), (2.9) is at most

$$
\exp \left[c z-c(1-c) \frac{B}{m-1} \sum_{i=1}^{m-1} \frac{g\left(s_{i} / v\right) s_{i}^{\alpha}}{g(1 / v)}\right] .
$$

Since $g$ is monotonic, this is dominated by

$$
\exp \left[c z-\frac{c(1-c) B}{m-1} \sum_{i=1}^{m-1} A\left(s_{i}\right)\right]
$$

where $A(s)=s^{\alpha}$ or 0 accordingly as $s>1$ or $s \leqq 1$. When multiplied by $e^{-z}$, the function (2.11) is integrable over $z, s_{1}, \ldots, s_{m-1}$ and dominates the integrand in (2.7). This justifies the passage to the limit under the integral sign. The proof is complete.

The following is a preliminary result which will later be improved.

LEMMA 2.1. Under the condition (0.2), for every $T>0$,

$$
\limsup _{u \rightarrow \infty} \frac{P\{\max (X(s): 0 \leqq s \leqq T)>u\}}{T v \phi(u) / u}<\infty .
$$

Proof. The interval $[0, T]$ is divisible into approximately $T v$ intervals, of each length $1 / v$; thus, by Boole's inequality and stationarity, it suffices to show that

$$
\limsup _{u \rightarrow \infty} \frac{P\{\max (X(s): 0 \leqq s \leqq 1 / v)>u\}}{\phi(u) / u}<\infty .
$$


Since

$$
P\{\max X(s)>u\} \leqq P\{X(0)>u\}+P\{\max X(s)>u, X(0) \leqq u\},
$$

it suffices, by the well-known estimate

$$
\phi(u)\left(\frac{1}{u}-\frac{1}{u^{3}}\right) \leqq \int_{u}^{\infty} \phi(x) d y \leqq \frac{\phi(u)}{u},
$$

to show that the lim sup of

$$
P\{\max (X(s / v): 0 \leqq s \leqq 1)>u, X(0) \leqq u\} /(\phi(u) / u)
$$

is finite. By the total probability formula this is equal to

$$
\frac{u}{\phi(u)} \int_{-\infty}^{u} P\left\{\max \left(X\left(\frac{s}{v}\right): 0 \leqq s \leqq 1\right)>u \mid X(0)=y\right\} \phi(y) d y,
$$

which, as in the argument leading to (2.3), is equal to

$$
\int_{0}^{\infty} P\left\{\max _{0 \leqq s \leqq 1} u\left[X\left(\frac{s}{v}\right)-u\right]>0 \mid X(0)=u-\frac{z}{u}\right\} \exp \left[z-z^{2} / 2 u^{2}\right] d z .
$$

By formula (2.4), with $-z$ in place of $z$, the conditional mean of $u[X(s / v)-u]$ is

$$
u^{2}[r(s / v)-1]-r(s / v) z \text {. }
$$

Put

$$
X_{u}(s)=u^{2}[X(s / v)-u]-u^{2}[r(s / v)-1]+r(s / v) z
$$

Now $r(s / v) \rightarrow 1$ as $u \rightarrow \infty$ uniformly for $0 \leqq s \leqq 1$; furthermore, we are concerned only with large values of $u$ in proving the lemma; thus we may suppose that the conditional mean (2.14) satisfies

$$
u^{2}[r(s / v)-1]-r(s / v) z<-\frac{1}{2} z, \text { for } 0 \leqq s \leqq 1, \text { and } z \geqq 0 .
$$

It follows that the conditional probability in (2.13) is at most

$$
P\left\{\max _{0 \leqq s \leqq 1} X_{u}(s)>\frac{1}{2} z \mid X(0)=u-\frac{z}{u}\right\} .
$$

By (2.14) and the definition of $X_{u}$,

$$
\begin{aligned}
E\left[X_{u}(s) \mid X(0)\right. & =u-z / u] \equiv 0 ; \\
E\left\{\left|X_{u}(t)-X_{u}(s)\right|^{2} \mid X(0)=u-z / u\right\} & =\operatorname{Var}\left\{\left[X_{u}(t)-X_{u}(s)\right]|X(0)=u-z| u\right\} \\
& \leqq \operatorname{Var}\left(X_{u}(t)-X_{u}(s)\right) \\
& =u^{2} E|X(t / v)-X(s / v)|^{2} \\
& =2 u^{2}[1-r((t-s) / v)], \quad 0<s<t .
\end{aligned}
$$


By (0.1) and (0.2) there exists a constant $K>0$ such that

$$
2 u^{2}[1-r((t-s) / v)] \leqq K|t-s|^{\alpha}, \quad 0<s<t \leqq 1,
$$

and where $K$ does not depend on $u$. We now apply Fernique's inequality [7] (for the proof, see [9]) to the conditioned process $X_{u}$ : we find that (2.15) is at most

$$
\text { constant } \times \int_{z / \text { constant }}^{\infty} \phi(y) d y \text {. }
$$

Put this bound in (2.13), and use the estimate (2.12); then (2.13) is bounded.

LEMMA 2.2. Let $U(t), t \geqq 0$, be the process defined before Theorem 2.1. For every $z>0$ and every $\tau$,

$$
E\left\{\exp \left(\tau \int_{0}^{\infty} I_{\left[U(t)>t^{\alpha}-z\right]} d t\right)\right\} \leqq \int_{0}^{\infty} \exp \left[\tau(\sqrt{ } 2 y+\sqrt{ } z)^{\alpha / 2}\right] \phi(y) d y .
$$

The latter integral is finite.

Proof. Let $Y$ be a random variable with a standard normal distribution. The stochastic process $\sqrt{ } 2 Y t^{\alpha / 2}, t \geqq 0$, is Gaussian with mean 0 and covariance function $2(s t)^{\alpha / 2}$. We have

$$
\operatorname{Var}\left(\sqrt{ } 2 Y t^{\alpha / 2}\right)=\operatorname{Var} U(t)=2 t^{\alpha}
$$

and

$$
\begin{aligned}
E U(s) U(t) & =s^{\alpha}+t^{\alpha}-(t-s)^{\alpha} \\
& =\left(t^{\alpha / 2}-s^{\alpha / 2}\right)^{2}+2(s t)^{\alpha / 2}-(t-s)^{\alpha} \\
& \leqq 2(s t)^{\alpha / 2}, \quad 0<s<t
\end{aligned}
$$

(because $t^{\alpha / 2} \leqq s^{\alpha / 2}+(t-s)^{\alpha / 2}$ for $\left.0<s<t\right)$; thus,

$$
E U(s) U(t) \leqq E\left[\left(\sqrt{ } 2 Y s^{\alpha / 2}\right)\left(\sqrt{ } 2 Y t^{\alpha / 2}\right)\right], \quad s \neq t .
$$

We apply the well-known inequality of Slepian [12]: If $t_{1}, \ldots, t_{m}$ and $z$ are arbitrary real numbers, then

$$
P\left\{U\left(t_{i}\right)>t_{i}^{\alpha}-z, i=1, \ldots, m\right\} \leqq P\left\{\sqrt{ } 2 Y t_{i}^{\alpha / 2}>t_{i}^{\alpha}-z, i=1, \ldots, m\right\} .
$$

Integrate over $t_{i} \geqq 0, i=1, \ldots, m$; then

$$
E\left\{\int_{0}^{\infty} I_{\left[U(t)>t^{\alpha}-z\right]} d t\right\}^{m} \leqq E\left\{\int_{0}^{\infty} I_{\left[\sqrt{ } 2 Y t^{\alpha / 2}>t^{\alpha}-z\right]} d t\right\}^{m}
$$

The latter may be written as

$$
E\left\{\int_{0}^{\infty} I_{\left[\sqrt{ } 2 Y t>t^{2}-z\right]} d\left(t^{\alpha / 2}\right)\right\}^{m} .
$$


If $t>\sqrt{ } 2 Y^{+}+\sqrt{ } z$, then $\sqrt{ } 2 Y t<t^{2}-z$ because $\sqrt{ } 2 Y^{+}+\sqrt{ } z$ is larger than the roots of the equation $t^{2}-\sqrt{ } 2 Y t-z=0$; therefore,

$$
\int_{0}^{\infty} I_{\left[\sqrt{ } 2 Y t>t^{2}-z\right]} d\left(t^{\alpha / 2}\right) \leqq\left(\sqrt{ } 2 Y^{+}+\sqrt{ } z\right)^{\alpha / 2} ;
$$

consequently, the expectation (2.17) is not more than $E\left(\sqrt{ } 2 Y^{+}+\sqrt{ } z\right)^{m \alpha / 2}$. Now multiply by $\tau^{m} / m !$, and sum over $m \geqq 0$; (2.16) follows.

THEOREM 2.2. There exists a unique (up to an additive constant) bounded nondecreasing function $H_{\alpha}(x)$ on $(0, \infty)$ such that

$$
\int_{0}^{\infty} x^{m} d H_{\alpha}(x)=m \int_{0}^{\infty} E\left[\int_{0}^{\infty} I_{\left[U(t)>t^{\alpha}-z\right]} d t\right]^{m-1} e^{-z} d z
$$

for $m \geqq 1$, and

$$
\int_{0}^{\infty} e^{t x} d H_{\alpha}(x)<\infty, \quad t>0
$$

and

$$
H_{\alpha}(\infty)-H_{\alpha}(0+)>0 .
$$

Under (0.2) there also exists a positive constant $V_{\alpha}$ such that

$$
V_{\alpha}=\lim _{u \rightarrow \infty} \frac{P\{\max (X(s): 0 \leqq s \leqq T)>u\}}{T v \phi(u) / u} .
$$

Proof. Let $F_{u}(x), x \geqq 0$, be the distribution function of $v \int_{0}^{T} I_{[X(s)>u]} d s$. This random variable assumes a positive value if and only if $\max (X(s): 0 \leqq s \leqq T)>u$; therefore,

$$
F_{u}(\infty)-F_{u}(0+)=P\left\{\max _{0 \leqq s \leqq T} X(s)>u\right\}
$$

By Lemma 2.1, $\left(F_{u}(\infty)-F_{u}(0+)\right) /(T v \phi(u) / u)$ is bounded for $u \geqq 1$. By Theorem 2.1 the $m$ th moment of the function $\left(F_{u}(x)-F_{u}(0+)\right) /(T v \phi(u) / u)$ converges to the righthand side of (2.1). The moment generating function of the limiting moment sequence is finite everywhere: when multiplied by $t^{m} / m$ ! and summed over $m \geqq 1$, the right side of $(2.1)$ becomes

$$
t \int_{0}^{\infty} E\left\{\exp \left(t \int_{0}^{\infty} I_{\left[U(s)>s^{\alpha}-z\right]} d s\right)\right\} e^{-z} d z,
$$

which, by Lemma 2.2, is finite. The moment convergence theorem now implies that there exists a unique $H_{\alpha}$ such that

$$
\frac{\int_{0}^{\infty} x^{m} d F_{u}(x)}{T v \phi(u) / u} \rightarrow \int_{0}^{\infty} x^{m} d H_{\alpha}(x)
$$

In the particular case $m=1$, we have $\int_{0}^{\infty} x d H_{\alpha}(x)=1$. This affirms (2.18), (2.19), and (2.20). 
To prove (2.21) we show that

$$
\lim _{u \rightarrow \infty} \frac{F_{u}(\infty)-F_{u}(0+)}{T v \phi(u) / u}=H_{\alpha}(\infty)-H_{\alpha}(0+) .
$$

The relation (2.24) implies the weak convergence of $d F_{u} / T v \phi(u) / u$ to $d H_{\alpha}$ on the open interval $(0, \infty)$, and also complete convergence on $(0, \infty]$ :

$$
\lim _{u \rightarrow \infty} \frac{F_{u}(\infty)-F_{u}(a)}{T v \phi(u) / u}=H_{\alpha}(\infty)-H_{\alpha}(a) \text { for } a>0 .
$$

This implies "half" of (2.25):

$$
\liminf _{u \rightarrow \infty} \frac{F_{u}(\infty)-F_{u}(0+)}{T v \phi(u) / u} \geqq H_{\alpha}(\infty)-H_{\alpha}(0+) .
$$

For arbitrary $x>0$ replace $u$ by $u+x / u$; then $v$ is still the "right" function of $u$ for large $u$ (cf. opening paragraph of $\S 3$ below), and so

$$
\limsup _{u \rightarrow \infty} \frac{F_{u+x / u}(\infty)-F_{u+x / u}(0+)}{T v \phi(u+x / u) /(u+x / u)}=\limsup _{u \rightarrow \infty} \frac{F_{u}(\infty)-F_{u}(0+)}{T v \phi(u) / u} \text {. }
$$

From this and the relation $\phi(u+x / u) \sim \phi(u) e^{-x}$, we get

$$
\limsup _{u \rightarrow \infty} \frac{F_{u+x / u}(\infty)-F_{u+x / u}(0+)}{T v \phi(u) / u}=e^{-x} \limsup _{u \rightarrow \infty} \frac{F_{u}(\infty)-F_{u}(0+)}{T v \phi(u) / u} .
$$

Note the identity

$$
\begin{aligned}
& F_{u+x / u}(\infty)-F_{u+x / u}(0+) \\
& \quad=P\left\{\max _{[0, T]} X>u+x / u, v L(u) \leqq \varepsilon\right\}+P\left\{\max _{[0, T]} X>u+x / u, v L(u)>\varepsilon\right\},
\end{aligned}
$$

and (2.26) and (2.28); then

$$
\begin{aligned}
\limsup _{u \rightarrow \infty} & e^{-x} \frac{F_{u}(\infty)-F_{u}(0+)}{T v \phi(u) / u} \\
\quad= & \limsup _{u \rightarrow \infty} \frac{P\left\{\max _{[0, T]} X>u+x / u, v L(u) \leqq \varepsilon\right\}}{T v \phi(u) / u}+H_{\alpha}(\infty)-H_{\alpha}(\varepsilon) .
\end{aligned}
$$

We shall show that the first term on the right-hand side tends to 0 with $\varepsilon$ for every $x>0$; hence, since $x$ is arbitrary, it will follow that

$$
\limsup _{u \rightarrow \infty} \frac{F_{u}(\infty)-F_{u}(0+)}{T v \phi(u) / u} \leqq H_{\alpha}(\infty)-H_{\alpha}(0+) .
$$

Combining this with (2.27) we get (2.25).

To complete the proof we estimate the first term on the right-hand side of (2.29). For simplicity put $T=1$. The unit interval is divisible into approximately $v$ subintervals of length $1 / v$; furthermore, it is evident that

$$
v \int_{A} I_{[X(s)>u]} d s \leqq v L(u)
$$


for every subinterval $A$ of $[0,1]$; thus, by the argument in the proof of Lemma 2.1 (Boole's inequality and stationarity) we find that

is at most equal to

$$
P\left\{\max _{[0,1]} X>u+x / u, v L(u) \leqq \varepsilon\right\}
$$

$$
v P\left\{\max _{0 \leqq s \leqq 1} u(X(s / v)-u)>x \int_{0}^{1} I_{[u(X(s / v)-u)>0]} d s \leqq \varepsilon\right\} .
$$

Now write the probability as the integral of the conditional probability given $X(0)=u-z / u$. By the standard weak convergence methods ([15], those based on the estimates of the conditional moments of the increments of the process, as in the proof of Lemma 2.1) it can be shown that the conditioned process $u(X(s / v)-u)$, $0 \leqq s \leqq 1$, converges not only in distribution to the process $U(s)-s^{\alpha}-z$, as in Theorem 2.1 , but also weakly over the function space $C[0,1]$; therefore, the joint distribution of the functionals in (2.30) (the maximum and the occupation time) converges to the joint distribution under the limiting process. Divide (2.30) by $\phi(u) / u$ and pass to the limit under the conditional expectation integral, as in Lemma 2.1 ; the ratio converges to

$$
\int_{0}^{\infty} P\left\{\max _{[0,1]} U(s)-s^{\alpha}>x+z, \int_{0}^{1} I_{\left[U(s)-s^{\alpha}>z\right]} d s \leqq \varepsilon\right\} e^{z} d z .
$$

The integrand converges pointwise to 0 as $\varepsilon \rightarrow 0$; indeed if the process $U(s)-s^{\alpha}$ spends little time above $z$, then it is very unlikely to exceed $z+x$. Passage to the limit under the sign of integration is again permitted by Fernique's inequality. The proof is complete.

An immediate consequence of Theorems 2.1 and 2.2 is:

THEOREM 2.3. Let $\Psi_{\alpha}$ be the distribution function with the moment sequence

$$
V_{\alpha}^{-1} \int_{0}^{\infty} x^{m} d H_{\alpha}(x), \quad m=1,
$$

that is, the distribution defined as

$$
\begin{aligned}
0 & \text { for } x \leqq 0 \\
\left(H_{\alpha}(x)-H_{\alpha}(0+)\right) / V_{\alpha} & \text { for } x>0 .
\end{aligned}
$$

Under (0.2), the conditional distribution of $v \int_{0}^{T} I_{[X(s)>u]} d s$, given that it is positive, converges to $\Psi_{\alpha}$.

Proof. For any nonnegative random variable $Y, E\left(Y^{m} \mid Y>0\right)=E Y^{m} / P\{Y>0\}$; thus, by (2.22),

$$
E\left\{\left[v \int_{0}^{T} I_{[X(s)>u]} d s\right]^{m} \mid v \int_{0}^{T} I_{[X(s)>u]} d s>0\right\}=\frac{E\left\{v \int_{0}^{T} I_{[X(s)>u]} d s\right\}^{m}}{P\{\max (X(s): 0 \leqq s \leqq T)>u\}}
$$

By Theorems 2.1 and 2.2 this converges, for $m \geqq 1$, to the right-hand side of (2.1), divided by $V_{\alpha}$. This is the $m$ th moment of the distribution function $\Psi_{\alpha}$. Since $H_{\alpha}$ 
has a moment generating function which is everywhere finite, so does $\Psi_{\alpha}$; thus, $\Psi_{\alpha}$ is uniquely determined by its moments. The assertion of the theorem now follows by application of the moment convergence theorem.

3. Limiting distribution of the maximum. We begin with some remarks about the construction of the function $v=v(u)$ in (0.3). This condition was used in the proofs in $\$ 2$ only to show that $u^{2}[1-r(s / v)]$ converges to $s^{\alpha}$ for $u \rightarrow \infty$. If $w(u)$ is an increasing function of $u$ such that $w(u) / u \rightarrow 1$ for $u \rightarrow \infty$, and if $v^{\prime}=v^{\prime}(w)$ is defined as

$$
w^{2} g\left(1 / v^{\prime}\right)\left(v^{\prime}\right)^{-\alpha}=1,
$$

then $u^{2}\left[1-r\left(s / v^{\prime}\right)\right] \sim w^{2}\left[1-r\left(s / v^{\prime}\right)\right] \rightarrow s^{\alpha}$. So $v^{\prime}$ may be interchanged with $v$; in other words, $v$ may be defined by $(0.3)$ not only in terms of $u$ but also in terms of any function asymptotic to $u$.

In this section we consider the limiting distribution of $\max (X(s): 0 \leqq s \leqq t)$ for $t \rightarrow \infty$. For $t>0$, let $v$ be defined in terms of $t$ as the unique solution of

$$
2 \log t g(1 / v) v^{-\alpha}=1 \text {. }
$$

Our result is

THEOREM 3.1. If (0.2) and (0.5) hold, then the random variable

$$
\sqrt{ }(2 \log t)\left[\max _{0 \leqq s \leqq t} X(s)-\sqrt{ }(2 \log t)-\frac{\log \left(v V_{\alpha} / 2 \sqrt{ }(\pi \log t)\right)}{\sqrt{ }(2 \log t)}\right]
$$

has, for $t \rightarrow \infty$, the limiting distribution $\exp \left(-e^{-x}\right)$.

(The constant $V_{\alpha}$ is defined in Theorem 2.2.)

Proof. The main idea of the proof is, as in previous studies, that the distribution of the maximum of the process over a large time interval is close to the distribution of the maximum of certain independent random variables. Suppose $t$ is a positive integer; then

$$
\max _{0 \leqq s \leqq t} X(s)=\max _{1 \leqq j \leqq t}\left(\max _{j-1 \leqq s \leqq j} X(s)\right) .
$$

If $\max (X(s): j-1 \leqq s \leqq j), j=1, \ldots, t$, were independent, then $\max (X(s): 0 \leqq s \leqq t)$ would be the maximum of $t$ independent random variables with the common distribution function

$$
G(x)=P\left\{\max _{0 \leqq s \leqq 1} X(s) \leqq x\right\} ;
$$

thus, $\max (X(s): 0 \leqq s \leqq t)$ would have the distribution function $G^{t}(x)$.

For fixed $x$, put

$$
u=\sqrt{ }(2 \log t)+\frac{\left(\log \left(v V_{\alpha} / 2 \sqrt{ }(\pi \log t)\right)+x\right)}{\sqrt{ }(2 \log t)} .
$$


By the definition (3.1) of $v$, and the increasing character of $g$, we have $v^{\alpha} / 2 \log t \rightarrow g(0)$ (positive or zero); therefore,

$$
\left(\log \left(v V_{\alpha} / 2 \sqrt{ }(\pi \log t)\right)\right) / \sqrt{ }(2 \log t) \rightarrow 0,
$$

and so, by (3.2),

$$
u \sim \sqrt{ }(2 \log t), \quad t \rightarrow \infty .
$$

The opening remarks of this section imply: If $v$ is defined by (3.1) and $u$ by (3.2), then the relations between $u$ and $v$ employed in $\$ 2$ may also be used under these new definitions.

It follows from (2.21) (with $T=1$ ), (3.2) and (3.3) that $1-G(u) \sim e^{-x} t^{-1}, t \rightarrow \infty$; therefore,

$$
G^{t}(u) \rightarrow \exp \left(-e^{-x}\right)
$$

thus, the limiting distribution of the maximum of $t$ independent random variables with the common distribution function $G$ is $\exp \left(-e^{-x}\right)$.

The rest of the proof consists of showing that the submaxima over the various intervals may actually be assumed to be asymptotically independent. The proof follows a familiar pattern; we shall, wherever possible, refer to previous work for details.

We break the interval $[0, t]$ into approximately $[t]$ intervals of unit length. (There is a small piece left over when $t$ is not an integer; however, the proof for such $t$ is reducible to that for integral $t$.) For arbitrary $\varepsilon, 0<\varepsilon<1$, we clip an open segment of length $\varepsilon$ from the right endpoint of each interval. The remaining intervals are $I_{j}=[j-1, j-\varepsilon], j=1, \ldots,[t]$. We have

$$
\limsup _{t \rightarrow \infty}\left|P\left\{\max _{0 \leqq s \leqq t} X(s)>u\right\}-P\left\{\max _{I_{1} \cup \cdots \cup I_{[t]}} X(s)>u\right\}\right| \leqq \varepsilon e^{-x}
$$

in fact, by Boole's inequality and stationarity, the difference of probabilities in (3.5) is not more than $t P\{\max (X(s): 0 \leqq s \leqq \varepsilon)>u\}$, which, by (2.21) and (3.2), converges to $\varepsilon e^{-x}$.

Let $M_{t}$ be the set of integer multiples of $(\log t)^{-3 / \alpha}$; then, by the argument in [10],

$$
\lim _{t \rightarrow 0} \sqrt{ }(2 \log t)\left|\max _{I_{1} \cup \cdots \cup I_{[t]}} X(s)-\max _{M_{t} \cap\left(I_{1} \cup \cdots \cup I_{[t])}\right.} X(s)\right|=0
$$

in probability; thus, the limiting distributions of the two maxima are the same. Put

$$
n=\text { integral part of } t(\log t)^{3 / \alpha},
$$

and let $\phi\left(u_{1}, u_{2} ; \rho\right)$ be the standard bivariate normal density with correlation coefficient $\rho$. 
We shall show that in calculating the limiting distribution of the maximum over $\left(I_{1} \cup \cdots \cup I_{[t]}\right) \cap M_{t}$, we may assume that the pieces of the process corresponding to different intervals $I_{j}$ are mutually independent. As in [4], it suffices to show that

$$
n \sum_{n \varepsilon / t \leq j \leqq n} \int_{0}^{r(j t / n)} \phi(u, u ; y) d y
$$

converges to 0 as $t \rightarrow \infty$.

By (0.5), the remark following (1.1), and the Riemann-Lebesgue lemma, it follows that $r^{p}(t) \rightarrow 0$ for $t \rightarrow \infty$; therefore, $r(t) \rightarrow 0$. By the same argument as in [4], it suffices to show: there exists $q, 0<q<1$, such that

$$
n t^{q-2} \sum_{j=1}^{n}|r(j t / n)| \rightarrow 0 \text { for } t \rightarrow \infty
$$

By the Hölder inequality,

$$
\sum_{j=1}^{n}|r(j t / n)| \leqq n^{1-(1 / 2 p)}\left(\sum_{j=1}^{n} r^{2 p}(j t / n)\right)^{1 / 2 p}
$$

Let $f^{(p)}(\lambda)$ be the Radon-Nikodým derivative of $F^{(p)}(\lambda)$; then, as is well known, the convolution $f^{(2 p)}(\lambda)$ is a continuous function, and

$$
r^{2 p}(j t / n)=2 \int_{0}^{\infty} \cos (\lambda j t / n) f^{(2 p)}(\lambda) d \lambda .
$$

Substitute this in (3.10); then, by the well-known cosine summation formula, the right-hand side of (3.10) is at most

$$
n^{1-(1 / 2 p)}\left(2 \int_{0}^{\infty} \frac{\sin \lambda t(1+1 / 2 n)}{\sin (\lambda t / 2 n)} f^{(2 p)}(\lambda) d \lambda\right)^{1 / 2 p}
$$

By a standard argument and the relation $t / n \rightarrow 0$, the last expression is asymptotic to

$$
n\left(4 \int_{0}^{\infty} \frac{\sin \lambda t}{\lambda t} f^{(2 p)}(\lambda) d \lambda\right)^{1 / 2 p} .
$$

For arbitrary $\beta, 0<\beta<1$, this is at most (by $|\sin \lambda t| \leqq|\lambda t|^{\beta}$ )

$$
n\left(4 t^{\beta-1} \int_{0}^{\infty} \lambda^{\beta-1} f^{(2 p)}(\lambda) d \lambda\right)^{1 / 2 p}
$$

The integral is finite because $f^{(2 p)}$ is continuous; thus, the expression above is of the order $n t^{(\beta-1) / p}$; therefore, the quantity on the left side of (3.9) is of the order $n^{2} t^{q-2+(\beta-1) / p}$. By the definition (3.7) of $n$, this converges to 0 if $q$ is chosen so that $0<q<(1-\beta) / p$; therefore, (3.9) holds.

By the remarks preceding (3.8) and (3.9), the distribution of the maximum on $M_{t} \cap\left(I_{1} \cup \ldots \cup I_{[t]}\right)$ is asymptotically the same as that of $[t]$ independent random variables with the common distribution function

$$
\bar{G}(x)=P\left\{\max _{M_{t} \cap I_{1}} X(s) \leqq x\right\} .
$$


By the same argument as for (3.6), we may replace $M_{t} \cap I_{j}$ by the full set $I_{j}$, $j=1, \ldots,[t]$; thus, the maximum is asymptotically the same as the maximum of $[t]$ independent random variables with the common distribution function

$$
G_{\varepsilon}(x)=P\left\{\max _{0 \leqq s \leqq 1-8} X(s) \leqq x\right\} .
$$

By the reasoning leading to (3.4), with $T=1-\varepsilon$, we find that the limiting distribution of $\max X(s)$ over $I_{1} \cup \cdots \cup I_{[t]}$ is $\exp \left(-(1-\varepsilon) e^{-x}\right)$. Since $\varepsilon>0$ is arbitrary, we put $\varepsilon=0$ here and in (3.5). This completes the proof of the theorem.

As in [4], we have

COROLlary 3.1. For arbitrary $b>0$, and with $u$ given by (3.2),

$$
\lim _{t \rightarrow \infty} P\left\{\max _{0 \leqq s \leqq t b} X(s) \leqq u\right\}=\exp \left(-b e^{-x}\right) .
$$

Another result is

COROLlaRY 3.2. If $I_{1}, I_{2}, \ldots$ are the intervals defined above, then

$$
\begin{aligned}
\sum_{i, j=1, i \neq j}^{[t]} \mid P\left\{\max _{I_{i}} X(s)>u, \max _{I_{j}} X(s)\right. & >u\} \\
& -P\left\{\max _{I_{i}} X(s)>u\right\} \cdot P\left\{\max _{I_{j}} X(s)>u\right\} \mid
\end{aligned}
$$

converges to 0 as $t \rightarrow \infty$.

Proof. By stationarity, the inequalities on $u$ may be reversed, and the summation simplified:

$$
\sum_{j=2}^{[t]}([t]-j)\left|P\left\{\max _{I_{1} \cup I_{j}} X(s) \leqq u\right\}-P^{2}\left\{\max _{I_{1}} X(s) \leqq u\right\}\right| .
$$

Let $M_{t}^{*}$ be the set of integral multiples of $(\log t)^{-6 / \alpha}$. By the same reasoning as for (3.6), the sets $I_{j}$ may be replaced by $I_{j} \cap M_{t}^{*}$. Put

$$
n=\text { integral part of } t(\log t)^{6 / \alpha},
$$

then, as in [4], the sum (3.11), with $I_{j} \cap M_{t}^{*}$ in place of $I_{j}, j=1, \ldots,[t]$, is at most

$$
\sum_{j=2}^{[t]}([t]-j) \cdot \frac{2 n}{t} \sum_{n \varepsilon / t \leqq k \leqq 2 n / t} \int_{0}^{r(k t / n+j-1)} \phi(u, u ; y) d y,
$$

which is at most

$$
2 n \sum_{n \varepsilon / t \leqq j \leqq 2 n} \int_{0}^{r(j t / n)} \phi(u, u ; y) d y .
$$

Like (3.8), it converges to 0 .

4. Preliminary estimates of the limiting distribution of the high level excursion. Throughout this section we assume that conditions $(0.2)$ and $(0.5)$ are satisfied. 
$v$ and $u$ are defined by (3.1) and (3.2), respectively. For fixed $\varepsilon, 0<\varepsilon<1, I_{j}$ is the interval $[j-1, j-\varepsilon]$, as defined in the previous section.

For fixed $b>0$, let $N$ be the number of intervals $I_{j}$ for which $\max \left(X(s): s \in I_{j}\right)$ $>u, j=1, \ldots,[t b]$. As the sum of $[t b]$ indicator random variables, $N$ has the expected value

$$
[t b] P\{\max (X(s): 0 \leqq s \leqq 1-\varepsilon)>u\}
$$

which, by Theorem 2.2, is asymptotic to $V_{\alpha} t b(1-\varepsilon) v \phi(u) / u$, which by (3.1) and (3.2), converges to $b(1-\varepsilon) e^{-x}$; hence

$$
E N \rightarrow b(1-\varepsilon) e^{-x}, \quad t \rightarrow \infty .
$$

By definition, $P\{N \geqq 1\}=P\left\{\max \left(X(s): s \in I_{1} \cup \cdots \cup I_{[t]}\right)>u\right\}$. By the proof of Theorem 3.1 and by Corollary 3.1, this implies

$$
P\{N \geqq 1\} \rightarrow 1-\exp \left[-b(1-\varepsilon) e^{-x}\right] .
$$

For any nonnegative integer valued random variable $N, P\{N>1\} \leqq E N-P\{N \geqq 1\}$; thus, by (4.1) and (4.2),

$$
\limsup _{t \rightarrow \infty} P\{N>1\} \leqq b(1-\varepsilon) e^{-x}-1+\exp \left[-b(1-\varepsilon) e^{-x}\right]
$$

therefore, from the inequality $e^{-w}-1+w \leqq \frac{1}{2} w^{2}, w>0$, it follows that

$$
\limsup _{t \rightarrow \infty} P\{N>1\} \leqq \frac{1}{2}\left[b(1-\varepsilon) e^{-x}\right]^{2} \leqq \frac{1}{2} b^{2} e^{-2 x} .
$$

LEMMA 4.1. Let $\Psi_{\alpha}$ be defined as in Theorem 2.3; then, for any $y>0$,

$$
\limsup _{t \rightarrow \infty} P\left\{0<v \sum_{j=1}^{[t b]} \int_{I_{j}} I_{[X(s)>u]} d s \leqq y\right\} \leqq b \Psi_{\alpha}(y) e^{-x}+\frac{1}{2} b^{2} e^{-2 x} .
$$

Proof. By the decomposition of the event into disjoint subsets, the probability in (4.4) is not more than

$$
P\left\{0<v \sum_{j=1}^{[t b]} \int_{I_{j}} I_{[X(s)>u]} d s \leqq y, N=1\right\}+P\{N>1\} .
$$

The events $\left\{v \int_{I_{j}} I_{[X(s)>u]} d s>0, N=1\right\}, j=1, \ldots,[t b]$, are disjoint; therefore, (4.5) is not more than

$$
\sum_{j=1}^{[t b]} P\left\{0<v \int_{I_{j}} I_{[X(s)>u]} d s \leqq y, N=1\right\}+P\{N>1\},
$$

which, by stationarity, is at most

$$
t b P\left\{0<v \int_{0}^{1-\varepsilon} I_{[X(s)>u]} d s \leqq y\right\}+P\{N>1\} .
$$


Now pass to the limit and apply Theorems 2.2 and 2.3, Corollary 3.1, and (4.3):

$$
\begin{aligned}
t b P\{0< & \left.v \int_{0}^{1-\varepsilon} I_{[X(s)>u]} d s \leqq y\right\} \\
& =t b P\left\{\max _{0 \leqq s \leqq 1-\varepsilon} X(s)>u\right\} \cdot P\left\{v \int_{0}^{1-\varepsilon} I_{[X(s)>u]} d s \leqq y \mid \max _{0 \leqq s \leqq 1-\varepsilon} X(s)>u\right\} \\
& \sim b e^{-x}(1-\varepsilon) \Psi_{\alpha}(y) .
\end{aligned}
$$

LEMMA 4.2. For $b>0$ and $y>0$,

$$
\liminf _{t \rightarrow \infty} P\left\{0<v \sum_{j=1}^{[t b]} \int_{I_{j}} I_{[X(s)>u]} d s \leqq y\right\} \geqq b(1-\varepsilon) e^{-x} \Psi_{\alpha}(y)-b^{2} e^{-2 x} .
$$

Proof. The probability above is at least

$$
P\left\{0<v \sum_{j=1}^{[t b]} \int_{I_{j}} I_{[X(s)>u]} d s \leqq y, N=1\right\},
$$

which, as in the previous proof, is equal to

$$
\sum_{j=1}^{[t b]} P\left\{0<v \int_{I_{j}} I_{[X(s)>u]} d s \leqq y, N=1\right\}
$$

This is equal to

$$
\sum_{j=1}^{[t b]} P\left\{0<v \int_{I_{j}} I_{[X(s)>u]} d s \leqq y, \max \left(X(s): s \in \bigcup_{k \neq j} I_{k}\right) \leqq u\right\},
$$

which is also equal to

$$
\begin{aligned}
\sum_{j=1}^{[t b]} P\{0<v & \left.\int_{I_{j}} I_{[X(s)>u]} d s \leqq y\right\} \\
& -\sum_{j=1}^{[t b]} P\left\{0<v \int_{I_{j}} I_{[X(s)>u]} d s \leqq y, \max \left(X(s): s \in \bigcup_{k \neq j} I_{k}\right)>u\right\} .
\end{aligned}
$$

By stationarity, Theorems 2.2 and 2.3, and (3.1) and (3.2), the first sum in (4.6) is asymptotic to

$$
t b P\left\{0<v \int_{0}^{1-\varepsilon} I_{[X(s)>u]} d s \leqq y\right\} \rightarrow b(1-\varepsilon) e^{-x} \Psi_{\alpha}(y) .
$$

The second sum in (4.6) is at most

$$
2 \sum_{j=1}^{[t b]} \sum_{k=j+1}^{[t b]} P\left\{\max _{I_{j}} X(s)>u, \max _{I_{k}} X(s)>u\right\}
$$

which, by Corollary 3.2 , is asymptotic to $[t b P\{\max (X(s): 0 \leqq s \leqq 1-\varepsilon)>u\}]^{2}$, which, by Theorem 2.2 , converges to $\left[b e^{-x}(1-\varepsilon)\right]^{2}<b^{2} e^{-2 x}$.

LEMMA 4.3. For $\lambda>0$, the Laplace-Stieltjes transform

$$
E\left[\exp \left(-\lambda v \sum_{j=1}^{[t b]} \int_{I_{j}} I_{[X(s)>u]} d s\right)\right]
$$


has a $\lim \sup (t \rightarrow \infty)$ not exceeding

$$
\exp \left[-b(1-\varepsilon) e^{-x}\right]+b e^{-x} \int_{0}^{\infty} e^{-\lambda y} d \Psi_{\alpha}(y)+\frac{b^{2} e^{-2 x}}{2 \lambda}
$$

and $a \lim$ inf at least equal to

$$
\exp \left[-b(1-\varepsilon) e^{-x}\right]+b e^{-x}(1-\varepsilon) \int_{0}^{\infty} e^{-\lambda y} d \Psi_{\alpha}(y)-\frac{b^{2} e^{-2 x}}{\lambda}
$$

Proof. Let $G(y)$ be the distribution function of

$$
v \sum_{j=1}^{[t b]} \int_{I_{j}} I_{[X(s)>u]} d s ;
$$

then, by the reasoning leading to (4.2),

$$
G(0+)=P\left\{\max \left(X(s): s \in \bigcup_{j=1}^{[t b]} I_{j}\right) \leqq u\right\} \rightarrow \exp \left[-b(1-\varepsilon) e^{-x}\right] .
$$

This and Lemmas 4.1 and 4.2 furnish bounds for the lim sup and lim inf of $G(y)$ $=[G(y)-G(0+)]+G(0+)$. The assertion of the lemma now follows by use of the identity

$$
\int_{0}^{\infty} e^{-\lambda y} d G(y)=1-\lambda \int_{0}^{\infty} e^{-\lambda y}[1-G(y)] d y .
$$

5. Limiting distribution of the time spent above $u$. If $X(t)$ satisfies $(0.6)$ then, as is well known, it has the stochastic integral representation

$$
X(s)=\int_{-\infty}^{\infty} e^{i \lambda s} \sqrt{ } f(\lambda) \xi(d \lambda)
$$

where $\xi$ is the standard Brownian motion process.

The function $r_{1}(s)=(1-|s|)^{+}$is a correlation function. Its spectral density is $(1-\cos \lambda) / \pi \lambda^{2}$. Put

$$
\rho(s)=\frac{\int_{-\infty}^{\infty} r_{1}(s-\tau) r_{1}(\tau) d \tau}{\int_{-\infty}^{\infty} r_{1}^{2}(\tau) d \tau}
$$

This is also a correlation function. Its spectral density is, by the convolution relation, equal to

$$
\varphi(\lambda)=\frac{\lambda^{-4}(1-\cos \lambda)^{2}}{\int_{-\infty}^{\infty} \lambda^{-4}(1-\cos \lambda)^{2} d \lambda}
$$

Since $r_{1}(s)$ vanishes outside $[-1,1], \rho(s)$ vanishes outside $[-2,2]$.

For $T>0$ we define a process $X_{T}(s)$ on the same probability space as the process $X(s)$ : Let $\xi$ be the Brownian motion in the representation (5.1), and put

$$
\left(\varphi_{T} f\right)(\lambda)=\int_{-\infty}^{\infty} f(\lambda+y / T) \varphi(y) d y
$$


then define $X_{T}$ as

$$
X_{T}(s)=\int_{-\infty}^{\infty} e^{i \lambda s} \sqrt{ }\left(\left(\varphi_{T} f\right)(\lambda)\right) \xi(d \lambda)
$$

This is also Gaussian and stationary with mean 0 , variance 1 and correlation function

$$
E X_{T}(0) X_{T}(s)=\int_{-\infty}^{\infty} e^{i \lambda s}\left(\varphi_{T} f\right)(\lambda) d \lambda=r(s) \rho(s / T),
$$

where $r(s)$ is the covariance function of $X(t)$ (cf. [2]).

LEMMA 5.1. If (0.7) holds, then

$$
\lim _{T \rightarrow \infty} \log T\left[1-E X(0) X_{T}(0)\right]=0 .
$$

Proof. It follows from (5.1) and (5.2) that

$$
E X(0) X_{T}(0)=\int_{-\infty}^{\infty}\left[f(\lambda) \cdot\left(\varphi_{T} f\right)(\lambda)\right]^{1 / 2} d \lambda
$$

therefore

$$
\begin{aligned}
1-E X(0) X_{T}(0) & =\int_{-\infty}^{\infty} \sqrt{ } f\left[\sqrt{ } f-\sqrt{ }\left(\varphi_{T} f\right)\right] d \lambda \\
& =\int_{-\infty}^{\infty} \frac{\sqrt{ } f}{\sqrt{ } f+\sqrt{ }\left(\varphi_{T} f\right)}\left(f-\left(\varphi_{T} f\right)\right) d \lambda \\
& \leqq \int_{-\infty}^{\infty}\left|f-\left(\varphi_{T} f\right)\right| d \lambda \leqq \int_{-\infty}^{\infty} \varphi(y)\left(\int_{-\infty}^{\infty}\left|f\left(\lambda+\frac{y}{T}\right)-f(\lambda)\right| d \lambda\right) d y .
\end{aligned}
$$

The last integral is evaluated by splitting the domain of integration with respect to $y$ :

$$
\int_{|y|<\log T} \varphi(y)\left(\int_{-\infty}^{\infty}\left|f\left(\lambda+\frac{y}{T}\right)-f(\lambda)\right| d \lambda\right) \leqq \sup _{y \leqq(\log T) / T} \int_{-\infty}^{\infty}|f(\lambda+y)-f(\lambda)| d \lambda .
$$

This is of smaller order than $(\log T)^{-1}$ for $T \rightarrow \infty$; indeed, if $h=(1 / T) \log T$, then $\log T \sim-\log h$, and so (0.7) implies

$$
-\log h \cdot \sup _{y \leqq h} \int_{-\infty}^{\infty}|f(\lambda+y)-f(\lambda)| d \lambda \leqq \sup _{y \leqq h}(-\log y) \int_{-\infty}^{\infty}|f(\lambda+y)-f(\lambda)| d \lambda \rightarrow 0 .
$$

The integral over the complementary domain is also $o\left((\log T)^{-1}\right)$ :

$$
\begin{aligned}
\int_{|y|>\log T} \varphi(y)\left(\int_{-\infty}^{\infty}\left|f\left(\lambda+\frac{y}{T}\right)-f(\lambda)\right| d \lambda\right) d y & \\
& \leqq \int_{|y|>\log T} \varphi(y)\left[2 \int_{-\infty}^{\infty} f(\lambda) d \lambda\right] d y=2 \int_{|y|>\log T} \varphi(y) d y .
\end{aligned}
$$

Since $\varphi(y)=O\left(\lambda^{-4}\right)$, the last integral, multiplied by $\log T$, converges to 0 . This completes the proof. 
Let $m$ be an arbitrary positive integer and $\delta$ an arbitrary real number, $0<\delta<1$. For $t>0$ decompose $[0, t]$ into $m$ equal subintervals of length $t / m$. Then clip off an open segment of length $t \delta / m$ from the right endpoint of each subinterval to form $m$ closed subintervals

$$
J_{k}=[(k-1) t / m,(k-\delta) t / m], \quad k=1, \ldots, m .
$$

Let $I_{j}$ be the intervals defined earlier and put $I=\bigcup_{j} I_{j}$.

LEMMA 5.2. Under (0.7) the random variables

$$
v \int_{J_{k} \cap I} I_{[X(s)>u]} d s, \quad k=1, \ldots, m,
$$

are asymptotically independent in the sense that the difference between their joint distribution and the product of their marginals converges to 0 as $t \rightarrow \infty$.

Proof. Put

$$
T=t \delta / 2 m ;
$$

then, by (5.3), $E X_{T}(s) X_{T}\left(s^{\prime}\right)=0$ if $\left|s-s^{\prime}\right|>t \delta / m$ because $\rho$ vanishes outside $[-2,2]$; therefore, the random variables $v \int_{J_{k} \cap I} I_{\left[X_{T}(s)>u\right]} d s, k=1, \ldots, m$, are mutually independent. For the proof of the lemma it is sufficient to show that

$$
\lim _{t \rightarrow \infty} E\left|v \int_{J_{k} \cap I} I_{[X(s)>u]} d s-v \int_{J_{k} \cap I} I_{\left[X_{T}(s)>u\right]} d s\right|=0, \quad k=1, \ldots, m .
$$

The expected absolute difference above is at most equal to

$$
2 v \int_{J_{k} \cap I} P\left\{X(s)>u, X_{T}(s) \leqq u\right\} d s,
$$

which, by stationarity, is

$$
2 v P\left\{X(0)>u, X_{T}(0) \leqq u\right\} \cdot \text { measure }\left(J_{k} \cap I\right)
$$

which is at most

$$
2 v P\left\{X(0)>u, X_{T}(0) \leqq u\right\} t / m .
$$

Put $\eta=E X(0) X_{T}(0)$; then, by an adaptation of the method in $[6$, p. 27],

$$
P\left\{X(0)>u, X_{T}(0) \leqq u\right\}=\int_{\eta}^{1} \phi(u, u ; y) d y .
$$

By the identity

$$
\phi(u, u ; y)=\phi(u) \phi\left(u /\left(\frac{1-y}{1+y}\right)\right)\left(1-y^{2}\right)^{-1 / 2}
$$

and the change of variable from $y$ to $u^{2}(1-y)$, we have

$$
\int_{\eta}^{1} \phi(u, u ; y) d y=\frac{\phi(u)}{u} \int_{0}^{u^{2}(1-\eta)} \phi\left(\sqrt{\left.\left(\frac{y}{2-y u^{-2}}\right)\right)\left[y\left(2-y u^{-2}\right)\right]^{-1 / 2} d y .}\right.
$$


By (3.2) this is asymptotic $(t \rightarrow \infty)$ to

$$
\frac{e^{-x}}{v t V_{\alpha}} \int_{0}^{(2 \log t)(1-\eta)} \phi(\sqrt{ }(y / 2)) \frac{d y}{\sqrt{ } y}
$$

It follows that (5.5) is asymptotic to

$$
\frac{2 e^{-x}}{V_{\alpha} m} \int_{0}^{(2 \log t)(1-\eta)} \phi(\sqrt{ }(y / 2)) \frac{d y}{\sqrt{ } y}
$$

which, by (5.4) and Lemma 5.1, converges to 0 as $t \rightarrow \infty$.

Our final result is

THEOREM 5.1. Under the assumptions $(0.2),(0.6)$ and $(0.7)$ the random variable

$$
v \int_{0}^{t} I_{[X(s)>u]} d s,
$$

where $u$ and $v$ are given by (3.2) and (3.1), has a limiting distribution whose LaplaceStieltjes transform is

$$
\exp \left\{e^{-x} \int_{0}^{\infty}\left(e^{-\lambda y}-1\right) d \Psi_{\alpha}(y)\right\}
$$

Proof. Put $b=(1-\delta) / m$; then

$$
v \int_{J_{1} \cap I} I_{[X(s)>u]} d s \text { and } v \sum_{j=1}^{[t b]} \int_{I_{j}} I_{[X(s)>u]} d s
$$

have the same limiting distributions because the difference between the two random variables is at most $v \int_{[t b]}^{t b} I_{[X[s]>u]} d s$, of which the expectation is at most $v P\{X(0)>u\}$, which, by (2.12), (3.1) and (3.2), converges to 0 . By Lemmas 4.3 and 5.2, the Laplace-Stieltjes transform of the sum

$$
\sum_{k=1}^{m} v \int_{J_{k} \cap I} I_{[X(s)>u]} d s
$$

has a lim sup at most equal to

$$
\left\{\exp \left[-e^{-x}(1-\delta)(1-\varepsilon) / m\right]+\frac{1-\delta}{m} e^{-x} \int_{0}^{\infty} e^{-\lambda y} d \Psi_{\alpha}(y)+\frac{(1-\delta)^{2}}{m^{2}} \cdot \frac{e^{-2 x}}{2 \lambda}\right\}^{m}
$$

and a lim inf at least equal to

$$
\left\{\exp \left[-e^{-x}(1-\delta)(1-\varepsilon) / m\right]+\frac{1-\delta}{m} e^{-x}(1-\varepsilon) \int_{0}^{\infty} e^{-\lambda y} d \Psi_{\alpha}(y)-\frac{(1-\delta)^{2} e^{-2 x}}{\lambda m^{2}}\right\}^{m} .
$$

Since $\bigcup_{k=1}^{m} J_{k} \cap I \subset[0, t]$, it follows from the relations (2.12), (3.1), and $\left|e^{-x}-e^{-x-h}\right| \leqq h$, for $x, h>0$, that

$$
\begin{aligned}
E\left|\exp \left[-\lambda v \sum_{k=1}^{m} \int_{J_{k} \cap I} I_{[X(s)>u]} d s\right]-\exp \left[-\lambda v \int_{0}^{t} I_{[X(s)>u]} d s\right]\right| \\
\leqq E\left|\lambda \sum_{k=1}^{m} v \int_{(k-\delta) t / m}^{k t / m} I_{[X(s)>u]} d s\right| \leqq \lambda t v \delta P\{X(0)>u\} \sim \lambda \delta / V_{\alpha} .
\end{aligned}
$$


It follows that the transform of (5.6) has a lim sup at most equal to expression (5.9) $+\lambda \delta / V_{\alpha}$ and a lim inf at least expression (5.10) $-\lambda \delta / V_{\alpha}$.

Since $\delta$ and $\varepsilon$ are arbitrary we put $\delta=\varepsilon=0$. Since $m$ is an arbitrary positive integer, we let $m \rightarrow \infty$ : The expressions (5.9) and (5.10), with $\delta=\varepsilon=0$, have the common limit (5.7).

REMARK. The transform (5.7) represents the distribution of the sum of a random number of independent random variables, where the summands have the common distribution $\Psi_{\alpha}$, and where the random number has a Poisson distribution with mean $e^{-x}$.

Added in proof. The validity of (2.21) under a weaker condition on $g$ was recently announced by C. R. Qualls and H. Watanabe in Notices Amer. Math. Soc. 18 (1971), 532. Abstract \#684-F4.

\section{REFERENCES}

1. Ju. K. Beljaev, On the number of level crossings by a Gaussian stochastic process. II, Teor. Verojatnost. i Primenen. 12 (1967), 444-457. (Russian) MR 36 \#948.

2. S. M. Berman, Occupation times of stationary Gaussian process, J. Appl. Probability 7 (1970), 721-733.

3. - Excursions above high levels for stationary Gaussian processes, Pacific J. Math. 36 (1971), 63-80.

4. - Asymptotic independence of the numbers of high and low level crossings of stationary Gaussian processes, Ann. Math. Statist. (to appear).

5. - - A class of limiting distributions of high level excursions of Gaussian processes, Z. Wahrscheinlichkeitstheorie und Verw. Gebiete (to appear).

6. H. Cramér and M. R. Leadbetter, Stationary and related stochastic processes. Sample function properties and their applications, Wiley, New York, 1967. MR 36 \#949.

7. X. Fernique, Continuité des processes Gaussiens, C. R. Acad. Sci. Paris 258 (1964), 60586060. MR 29 \#1662.

8. M. Loève, Probability theory, 3rd ed., Van Nostrand, Princeton, N. J., 1963. MR 34 \#3596.

9. M. B. Marcus, A bound for the distribution of the maximum of continuous Gaussian processes, Ann. Math. Statist. 41 (1970), 305-309.

10. J. Pickands III, Maxima of stationary Gaussian processes, Z. Wahrscheinlichkeitstheorie und Verw. Gebiete 7 (1967), 190-223. MR 36 \#955.

11. C. Qualls, On a limit distribution of high level crossings of a stationary Gaussian process, Ann. Math. Statist. 39 (1968), 2108-2113. MR 38 \#6661.

12. D. Slepian, The one-sided barrier problem for Gaussian noise, Bell System Tech. J. 41 (1962), 463-501. MR 24 \#A3017.

13. E. C. Titchmarsh, Introduction to the theory of Fourier integrals, 2nd ed., Clarendon Press, Oxford, 1948.

14. V. A. Volkonskiǐ and Ju. A. Rozanov, Some limit theorems for random functions. I, II. Teor. Verojatnost. i. Primenen. 4 (1959), 186-207=Theor. Probability Appl. 4 (1959), 178-197. MR 22 \#12586; Teor. Verojatnost. i Primenen. 6 (1961), 202-215. (Russian) MR 25 \#597.

15. P. Billingsley, Convergence of probability measures, Wiley, New York, 1968.

Courant Institute of Mathematical Sciences, New York University, New YoRK, New YoRK 10012 